\title{
Associated Production of Neutral Higgs Boson with Squark Pair in the Minimal Supersymmetric Standard Model with Explicit CP Violation at the CERN LHC
}

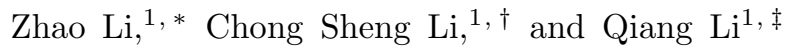 \\ ${ }^{1}$ Department of Physics, Peking University, Beijing 100871, China
}

\begin{abstract}
We investigate the associated production of neutral Higgs boson with squark pair in the minimal supersymmetric standard model with and without explicit CP violation, respectively. We show that the dominant productions in both cases are always ones of the lightest neutral Higgs boson associated with the lightest stop pair, which can reach a few pb, in the ranges of parameters allowed by constraints from the electric dipole moment experiments. In most of the parameter space, the total cross sections in the case with explicit $\mathrm{CP}$ violation are significantly enhanced, compared with ones without explicit CP violation. For some special parameters, several orders of magnitude enhancement can be obtained.
\end{abstract}

PACS numbers: 12.60.Jv, 14.80.Cp, 14.80.Ly

The minimal supersymmetric standard model (MSSM) with $\mathrm{CP}$ conservation (CPC) provides three neutral Higgs bosons: one $\mathrm{CP}$ odd boson $\left(A^{0}\right)$ and two $\mathrm{CP}$ even boson $\left(h^{0}, H^{0}\right)$, whose mass eigenstates are also their CP eigenstates. However, in the MSSM with explicit CP violation (CPV), $\mathrm{CP}$ violation can introduce the mixing of three neutral Higgs bosons, and CP eigenstates no longer exist. The mixing can form three new mass eigenstates of the neutral Higgs bosons: $h_{1}, h_{2}$ and $h_{3}$, and all couplings involved the neutral Higgs bosons are changed [1, 2]. The previous studies have shown that explicit $\mathrm{CP}$ violation mentioned above can significantly change the predictions for the Higgs productions and decays 1, 2, 3, 4]. Especially, Q. H. Cao et al. 4. recently found that there is a parameter region, in which the Higgs production cross sections via gluon fusion can be greatly enhanced with a large $\mathrm{CP}$ violation angle. However, in their calculations they did not consider the constraints on parameters from the experiments of electric dipole moments (EDMs), because they believe that there are possible cancelations between different contributions to EDMs.

In fact, the present EDM experiments [ 5,6 , 7, 8 ] have put some constraints on the parameter space in the MSSM with explicit CP violation in some scenarios. Therefore, it is necessary to make further investigation on the production of Higgs boson with a large CP violation angle, using the EDMs constraints on the relevant parameters. B- and K-physics may provide the constraints on parameters, but they depend strongly on the flavor structure in supersymmetry breaking [4]. Therefore, the constraints potentially arising from B- and K-physics are not considered in this letter.

In this letter, we first consider the EDM experiment constraints on the relevant parameters in the case of $\mathrm{CPV}$, and then using the allowed parameters, we investigate the neutral Higgs production associated with squark pair in the MSSM with and without explicit CP violation at the CERN Large Hadron Collider (LHC) 9, 10, 11, 12, 13, 14]. A similar process, the production of Higgs associated with squark pairs, has been calcu- lated in the minimal supergravity (mSUGRA) without CPV in Ref. 15.

Through the one-loop effective potential of the neutral Higgs sector, as shown in Ref. 2], the complex parameters in the mass matrices of sfermions can induce CPV in the Higgs sector. The input parameters of the mass matrix can be chosen as $m_{A}^{2}, A_{t} \mu \mathrm{e}^{\mathrm{i} \xi}$ and $A_{b} \mu \mathrm{e}^{\mathrm{i} \xi}$, and there are only two CP violation angles: $\kappa_{1}=\theta_{A_{t}}+\theta_{\mu}+\xi$ and $\kappa_{2}=\theta_{A_{b}}+\theta_{\mu}+\xi$. Therefore, it is equivalent that we choose $\theta_{\mu}=\xi=0$, and leave $\theta_{A_{t}}$ and $\theta_{A_{b}}$ as the $\mathrm{CP}$ violation angles. Diagonalizing the mass matrix of neutral Higgs, we get the mass eigenstates $h_{1}, h_{2}$ and $h_{3}$, and the corresponding mass eigenvalues $m_{h_{1}}, m_{h_{2}}$ and $m_{h_{3}}\left(m_{h_{1}} \leq m_{h_{2}} \leq m_{h_{3}}\right)$, which are different from the CP eigenstates, $\left(h^{0}, H^{0}, A^{0}\right)$. Using the relations between $\left(h^{0}, H^{0}\right)$ and $\left(\phi_{1}, \phi_{2}\right)$, we can obtain the relations between $\left(h^{0}, H^{0}, A^{0}\right)$ and $\left(h_{1}, h_{2}, h_{3}\right)$. The calculations of relevant processes considered here at the leading order are straightforward, so we do not show their expressions.

In our numerical calculations, the Standard Model (SM) parameters are chosen to be $\alpha_{e w}\left(m_{W}\right)=1 / 137$, $m_{Z}=91.1875 \mathrm{GeV}, m_{W}=80.45 \mathrm{GeV}$ and $m_{t}=$ $174 \mathrm{GeV}$ [16]. We use the CTEQ6M parton distribution functions (PDFs) 17] and the two-loop evaluation for $\alpha_{s}(Q)[18]\left(\alpha_{s}\left(m_{Z}\right)=0.118\right)$ and the running bottom quark mass 19. The other SM input parameters are 16, 20.

$$
\begin{array}{lll}
m_{e}=0.51 M e V, & m_{\mu}=105.658 \mathrm{MeV}, & m_{\tau}=1.777 \mathrm{GeV}, \\
m_{d}=4 \mathrm{MeV}, & m_{s}=120 \mathrm{MeV}, & m_{b}=4.2 \mathrm{GeV}, \\
m_{u}=2.5 \mathrm{MeV}, & m_{c}=1.2 \mathrm{GeV} . &
\end{array}
$$

In addition, we assume that the complex parameters only exist in the squark sector. For simplicity, we choose $A_{t}=$ $A_{b}=A_{u}=A_{d}=A e^{i \theta_{A}}$ and $m_{\tilde{Q}}=m_{\tilde{U}}=m_{\tilde{D}}=m_{\tilde{E}}=$ $m_{\tilde{L}}=M_{S U S Y}$. Then, using the solution of $m_{\tilde{t}_{1}}$, we can choose $m_{\tilde{t}_{1}}$ as input parameter instead of $M_{S U S Y}$. The other MSSM input parameters are fixed as following:

$$
\begin{aligned}
& M_{1}=100 \mathrm{GeV}, \quad M_{2}=200 \mathrm{GeV}, \quad M_{3}=300 \mathrm{GeV}, \\
& A_{l}=A_{\tau}=1000 \mathrm{GeV}, \quad \theta_{A}=\pi / 2, \quad \theta_{\mu}=\xi=0 .
\end{aligned}
$$


The remaining MSSM input parameters are $\tan \beta, M_{A^{0}}$, $m_{\tilde{t}_{1}}, A$ and $\mu$.

The strongest constraints on the parameters in the case of CPV arise from the experiments on the EDMs of thallium[ $[$ ], mercury [ $]$ ] and neutron [5]. Although it is supposed that cancelations possibly come from many contributions [4, 11, 14, 21], it is still necessary to investigate further the constraints on the parameter space in the case of explicit $\mathrm{CP}$ violation based on the recent detailed theoretical analyses $9,10,11,12,13,14$. The current experiments at LEPII and Tevatron have also set up the lower bounds 16, 20 on the masses of neutral Higgs, stop and sbottom, which are $89.8 \mathrm{GeV}, 95.7 \mathrm{GeV}$ and $89 \mathrm{GeV}$, respectively, and $\left|A_{t}\right|$ and $\left|A_{b}\right|$ can not be taken too large 15, 22 in order to avoid a color breaking vacuum expectation value (VEV).

Combining all the above constraints, we can obtain the allowed regions in the parameter space.

From the EDM expressions, we find that parameters spaces are constrained more stringently when $M_{A^{0}}$ becomes smaller. In order to calculate numerical results in larger ranges of parameters allowed by constraints from all experiments, we will consider the case of $M_{A^{0}}=$ $300 \mathrm{GeV}$ below.

Figs 12 show the allowed ranges of $A$ and $\mu$ for different $\tan \beta$ and $m_{\tilde{t}_{1}}$. In general, the constraints on both $A$ and $\mu$ become weaker for larger $m_{\tilde{t}_{1}}$, and the allowed ranges of $\mu$ are sharply constrained for large $\tan \beta$, especially for $\tan \beta=35$. We will mainly consider the case of $\tan \beta \geq 5$ in the following.

$\operatorname{Fig}\left[3\right.$ gives the allowed ranges of $\tan \beta$ and $m_{\tilde{t}_{1}}$ for different $A$ and $\mu$. $\tan \beta$ can be strongly constrained for larger $\mu$ and smaller $A$. For example, for $A=500 \mathrm{GeV}$ and $\mu=700 \mathrm{GeV}, \tan \beta$ is almost limited near 5 , and in the case of $\mu=700 \mathrm{GeV}, m_{\tilde{t}_{1}}$ is strongly constrained only for large $\tan \beta$.

Using the allowed ranges mentioned above, we investigate the productions of neutral Higgs associated with squark pair in the MSSM with and without explicit CP violation, respectively. In the experiments, the mass eigenstates of Higgs are the observables, which can be used to distinguish different Higgs bosons, and for comparison with the case of $\mathrm{CPC}$, we will mainly focus on the productions of the lightest Higgs associated with squark pairs. In the following, we define $\Phi=\left(h_{1}, h_{2}, h_{3}\right)$ for the case of CPV, and $\Phi=\left(h^{0}, H^{0}, A^{0}\right)$ for the case of CPC, respectively.

Fig 4 shows the total cross sections of two processes $p p \rightarrow \Phi_{1} \tilde{t}_{1} \tilde{t}_{1}^{*}\left(\Phi_{1}=h_{1}, h^{0}\right)$ and $p p \rightarrow \Phi_{1} \tilde{t}_{1} \tilde{t}_{2}^{*}$ as functions of $\tan \beta$, respectively, assuming $A=900 \mathrm{GeV}$, $\mu=400 \mathrm{GeV}, m_{\tilde{t}_{1}}=100 \mathrm{GeV}$ or $200 \mathrm{GeV}$. In both processes, the total cross sections in the case of $\mathrm{CPV}$ are larger than ones in the case of CPC, especially for $\tan \beta \approx 5$. The total cross sections for $\Phi_{1} \tilde{t}_{1} \tilde{t}_{1}^{*}$ produc- tions are the largest, which are about

$$
\sigma\left(h^{0} \tilde{t}_{1} \tilde{t}_{1}^{*}\right) \approx 2.55 p b \quad \text { and } \sigma\left(h_{1} \tilde{t}_{1} \tilde{t}_{1}^{*}\right) \approx 3.78 p b,
$$

respectively, for $A=900 \mathrm{GeV}, \mu=400 \mathrm{GeV}, m_{\tilde{t}_{1}}=$ $100 \mathrm{GeV}$ and $\tan \beta=5$. Note that $\sigma\left(h_{1} \tilde{t}_{1} \tilde{t}_{1}^{*}\right)$ are enhanced by about $50 \%$, compared with $\sigma\left(h^{0} \tilde{t}_{1} \tilde{t}_{1}^{*}\right)$. At the LHC, with an integrated luminosity of $100 \mathrm{fb}^{-1}$, we can expect that the events will be about $2.55 \times 10^{5}$ for $\sigma\left(h^{0} \tilde{t}_{1} \tilde{t}_{1}^{*}\right)$ and $3.78 \times 10^{5}$ for $\sigma\left(h_{1} \tilde{t}_{1} \tilde{t}_{1}^{*}\right)$, respectively, and those signals may be observable in future experiments. Fig 4 also shows that all the total cross sections for $m_{\tilde{t}_{1}}=200 \mathrm{GeV}$ are less than ones for $m_{\tilde{t}_{1}}=100 \mathrm{GeV}$.

In Fig [5] we plot the total cross sections of two processes $p p \rightarrow \Phi_{2} \tilde{t}_{1} \tilde{t}_{2}^{*}\left(\Phi_{2}=h_{2}, H^{0}\right)$ and $p p \rightarrow \Phi_{2} \tilde{b}_{1} \tilde{b}_{2}^{*}$ as functions of $\tan \beta$, respectively, assuming $A=900 \mathrm{GeV}$, $\mu=400 \mathrm{GeV}, m_{\tilde{t}_{1}}=100 \mathrm{GeV}$ or $200 \mathrm{GeV}$. Here we find that the total cross sections for $H^{0} \tilde{t}_{1} \tilde{t}_{1}^{*}$ production are larger than ones for $h_{2} \tilde{t}_{1} \tilde{t}_{1}^{*}$ production, and both can generally reach tens of fb.

For the associated productions of $A^{0} \tilde{q}_{i} \tilde{q}_{j}^{*}$ and $h_{3} \tilde{q}_{i} \tilde{q}_{j}^{*}$, the cross sections $\sigma\left(h_{3} \tilde{t}_{1} \tilde{t}_{1}^{*}\right), \sigma\left(h_{3} \tilde{t}_{1} \tilde{t}_{2}^{*}\right)$ and $\sigma\left(A^{0} \tilde{t}_{1} \tilde{t}_{2}^{*}\right)$ are all a few fb in general, and the other ones are all less than $1 \mathrm{fb}$.

Fig [6] shows the curves of $\sigma\left(\Phi_{1} \tilde{t}_{1} \tilde{t}_{1}^{*}\right)$ and $\sigma\left(\Phi_{1} \tilde{t}_{2} \tilde{t}_{2}^{*}\right)$ as functions of $A$, respectively, assuming $\tan \beta=5, m_{\tilde{t}_{1}}=$ $100 \mathrm{GeV}$ and $\mu=400 \mathrm{GeV}$ or $1000 \mathrm{GeV}$. The dominant processes are still $p p \rightarrow \Phi_{1} \tilde{t}_{1} \tilde{t}_{1}^{*}$. The total cross sections $\sigma\left(h_{1} \tilde{t}_{1} \tilde{t}_{1}^{*}\right)$ are enhanced, compared with $\sigma\left(h^{0} \tilde{t}_{1} \tilde{t}_{1}^{*}\right)$. And when $A>700 \mathrm{GeV}$, such enhancement increases with increasing $A$. Especially, the enhancement can approach to infinite for some special values of $A$ and $\mu$, because the total cross sections $\sigma\left(h^{0} \tilde{t}_{1} \tilde{t}_{1}^{*}\right)$ approach to zero then, because the squark-Higgs couplings tend to zero in the case of CPC. For example,

$$
\sigma\left(h^{0} \tilde{t}_{1} \tilde{t}_{1}^{*}\right)<10^{-3} f b, \quad \sigma\left(h_{1} \tilde{t}_{1} \tilde{t}_{1}^{*}\right) \approx 270 f b,
$$

for $A=568 \mathrm{GeV}$ and $\mu=1000 \mathrm{GeV}$.

Fig[7 gives the total cross sections of two processes $p p \rightarrow \Phi_{2} \tilde{t}_{1} \tilde{t}_{1}^{*}$ and $p p \rightarrow \Phi_{2} \tilde{t}_{2} \tilde{t}_{2}^{*}$ as functions of $A$, respectively, assuming $\tan \beta=5, m_{\tilde{t}_{1}}=100 \mathrm{GeV}$ and $\mu=400 \mathrm{GeV}$ or $1000 \mathrm{GeV}$. The total cross sections $\sigma\left(\Phi_{2} \tilde{t}_{1} \tilde{t}_{1}^{*}\right)$ can reach hundreds of $\mathrm{fb}$, corresponding to about $10^{4}$ events at the LHC, while the other ones are generally less than $1 \mathrm{fb}$.

For the processes $p p \rightarrow \Phi_{3} \tilde{q}_{i} \tilde{q}_{j}^{*}\left(\Phi_{3}=h_{3}, A^{0}\right)$, the total cross sections of $p p \rightarrow \Phi_{3} \tilde{t}_{1} \tilde{t}_{2}^{*}$ are the largest, which can reach hundreds of fb, but fastly decrease with increasing $A$. The other ones are less than a few $\mathrm{fb}$.

In conclusion, we have calculated the total cross sections of the neutral Higgs production associated with squark pair in the MSSM without and with explicit CP violation, respectively. The dominant processes in both cases are always $p p \rightarrow \Phi_{1} \tilde{t}_{1} \tilde{t}_{1}^{*}$, which can reach a few $\mathrm{pb}$, while the maximum results in mSUGRA without $\mathrm{CP}$ violation for the same final states are less than $500 \mathrm{fb}$ 
15]. In most of the parameter space, the total cross sections $\sigma\left(h_{1} \tilde{t}_{1} \tilde{t}_{1}^{*}\right)$ are always significantly enhanced, compared with $\sigma\left(h^{0} \tilde{t}_{1} \tilde{t}_{1}^{*}\right)$. For some special parameters, several orders of magnitude enhancement can be obtained.

This work was supported in part by the National Natural Science Foundation of China, under Grant Nos. 10421503 and 10575001, and the key Grant Project of Chinese Ministry of Education, under Grant No. 305001.

* Electronic address: zhli.phy@pku.edu.cn

† Electronic address: csli@pku.edu.cn

‡ Electronic address: qliphy@pku.edu.cn

[1] A. Pilaftsis and C. E. M. Wagner, Nucl. Phys. B553, 3 (1999).

[2] S. Y. Choi et al., Phys. Lett. B481, 57 (2000) and references therein.

[3] D. A. Demir, Phys. Rev. D60, 095007 (1999); A. Dedes and S. Moretti, Phys. Rev. Lett. 84, 22 (2000).

[4] Q.-H. Cao et al., Phys. Lett. B632, 688 (2006).

[5] P. G. Harris et al., Phys. Rev. Lett. 82, 904 (1999).

[6] B. C. Regan et al., Phys. Rev. Lett. 88, 071805 (2002).

[7] M. V. Romalis et al., Phys. Rev. Lett. 86, 2505 (2001).

[8] J. J. Hudson et al., Phys. Rev. Lett. 89, 023003 (2002).

[9] D. Chang et al., Phys. Lett. B478, 239 (2000).

[10] K. A. Olive et al., Phys. Rev. D72, 075001 (2005).

[11] T. Ibrahim and P. Nath, Phys. Rev. D57, 478 (1998).

[12] D. A. Demir et al., Nucl. Phys. B680, 339 (2004).

[13] A. Pilaftsis, Phys. Lett. B471, 174 (1999).

[14] T. Ibrahim and P. Nath, Phys. Rev. D58, 111301 (1998).

[15] A. Dedes and S. Moretti, Eur. Phys. J. C10, 515 (1999).

[16] CDF Collaboration, (2005), hep-ex/0512009.

[17] J. Pumplin et al., JHEP 07, 012 (2002).

[18] A. Djouadi, M. Spira, and P. M. Zerwas, Z. Phys. C70, 427 (1996).

[19] A. Djouadi et al., Comput. Phys. Commun. 108, 56 (1998).

[20] S. Eidelman et al. (Particle Data Group), Phys. Lett. B592, 1 (2004).

[21] J. R. Ellis et al., Phys. Rev. D72, 095006 (2005).

[22] J. A. Casas et al., Nucl. Phys. B471, 3 (1996).

Figures
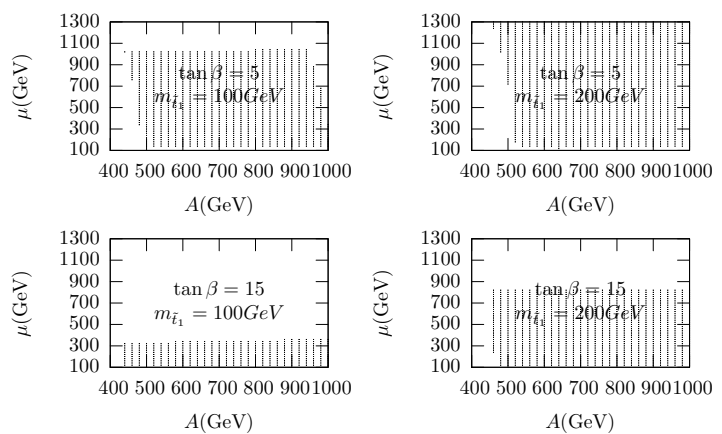

FIG. 1: The allowed ranges of the parameters $A$ and $\mu$ for $M_{A^{0}}=300 \mathrm{GeV}, \tan \beta=5$ and $15, m_{\tilde{t}_{1}}=100 \mathrm{GeV}$ and $200 \mathrm{GeV}$, respectively. The shaded areas correspond to the allowed ranges of parameters.
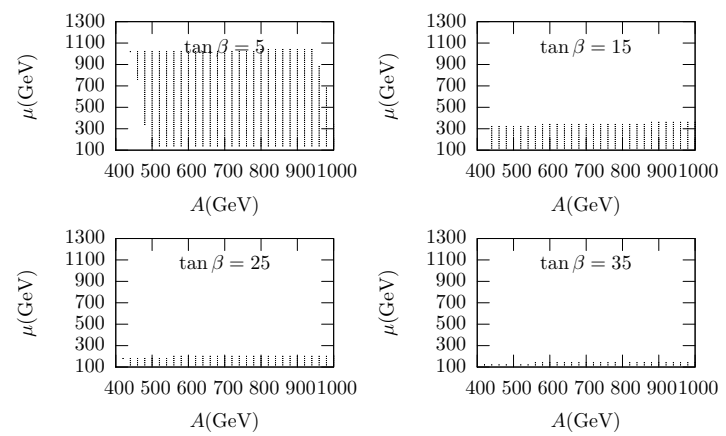

FIG. 2: The allowed ranges of the parameters $A$ and $\mu$ for $M_{A^{0}}=300 \mathrm{GeV}, m_{\tilde{t}_{1}}=100 \mathrm{GeV}, \tan \beta=5,15,25$ and 35 , respectively. The shaded areas correspond to the allowed ranges of parameters.
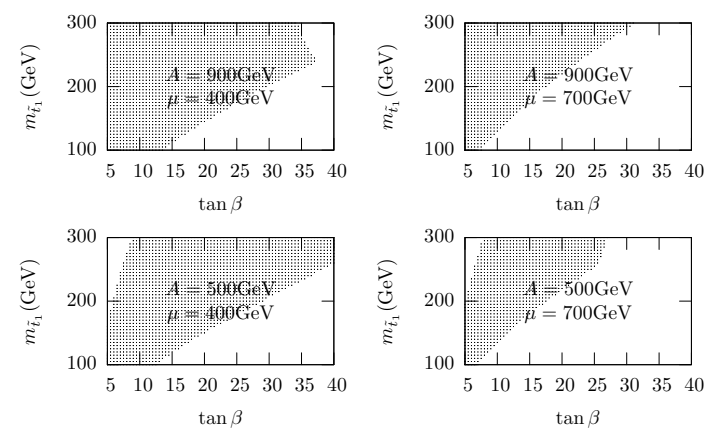

FIG. 3: The allowed ranges of the parameters $\tan \beta$ and $m_{\tilde{t}_{1}}$ for $M_{A^{0}}=300 \mathrm{GeV}, A=500 \mathrm{GeV}$ and $900 \mathrm{GeV}, \mu=400 \mathrm{GeV}$ and $700 \mathrm{GeV}$, respectively. The shaded areas correspond to the allowed ranges of parameters.

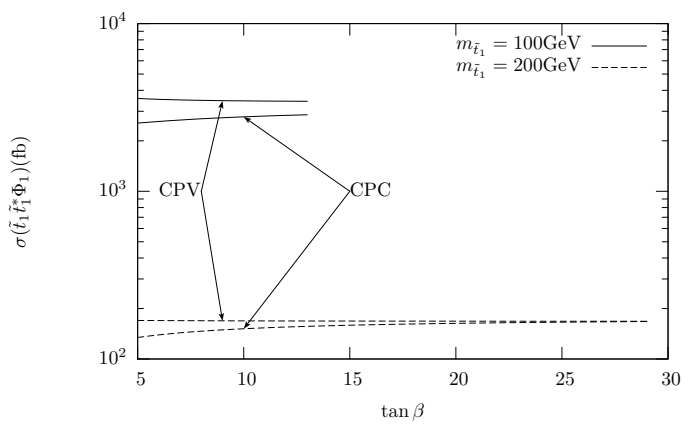




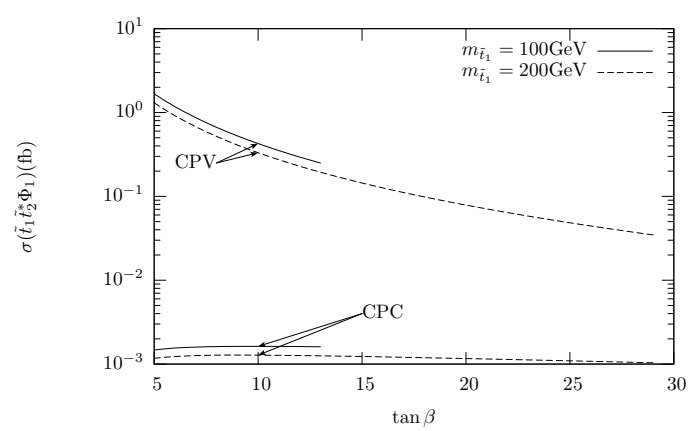

FIG. 4: The cross sections of $\Phi_{1}$ productions associated with squark pairs as the functions of $\tan \beta$, where $\Phi_{1}=h^{0}$ in $\mathrm{CPC}$ and $\Phi_{1}=h_{1}$ in $\mathrm{CPV}$, assuming $A=900 \mathrm{GeV}$ and $\mu=400 \mathrm{GeV}$.
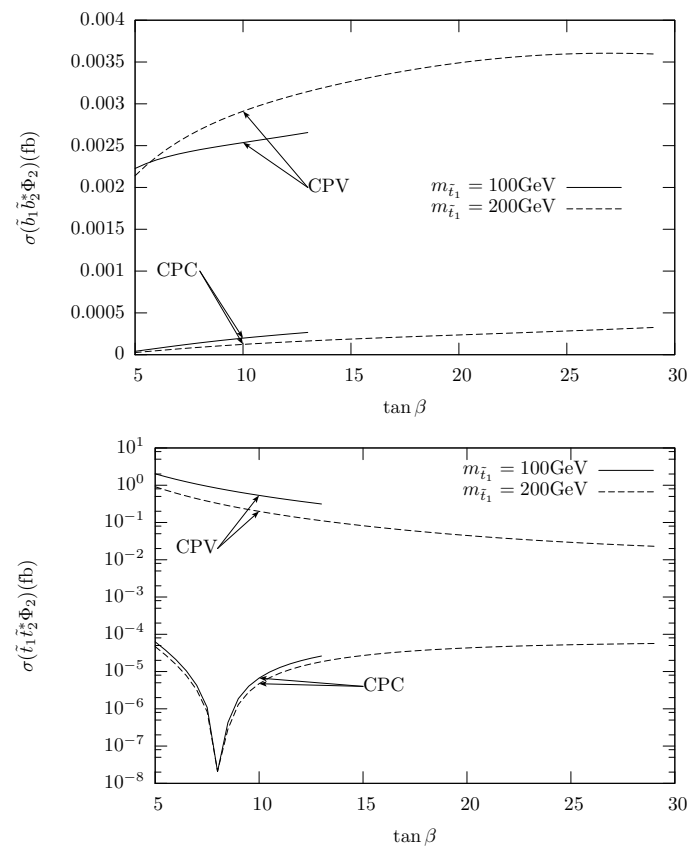

FIG. 5: The cross sections of $\Phi_{2}$ productions associated with squark pairs as the functions of $\tan \beta$, where $\Phi_{2}=H^{0}$ in $\mathrm{CPC}$ and $\Phi_{2}=h_{2}$ in $\mathrm{CPV}$, assuming $A=900 \mathrm{GeV}$ and $\mu=400 \mathrm{GeV}$.

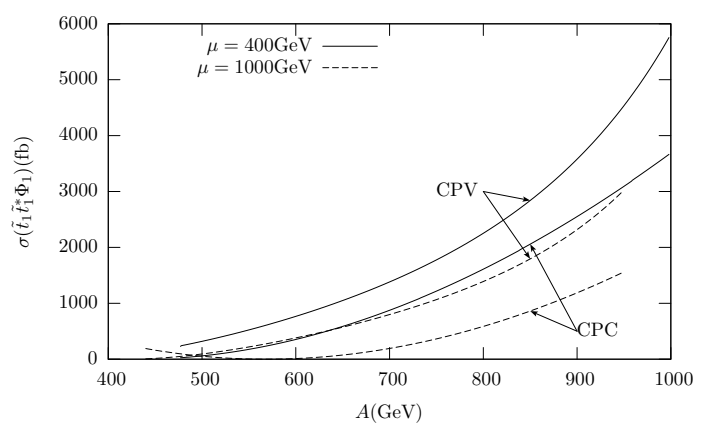

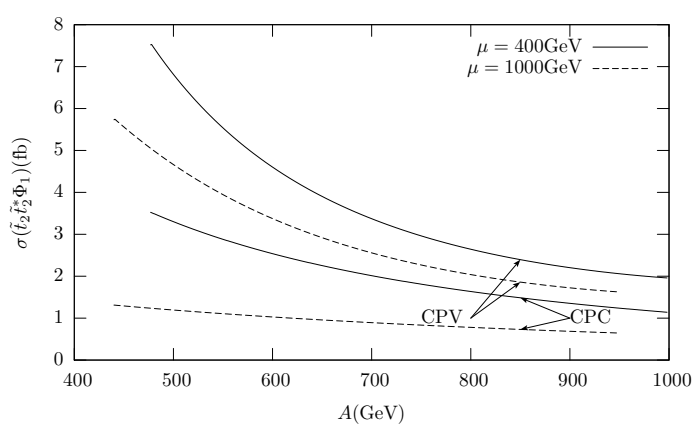

FIG. 6: The cross sections of $\Phi_{1}$ productions associated with squark pairs as the functions of $A$, where $\Phi_{1}=h^{0}$ in CPC and $\Phi_{1}=h_{1}$ in CPV, assuming $\tan \beta=5$ and $m_{\tilde{t}_{1}}=100 \mathrm{GeV}$.
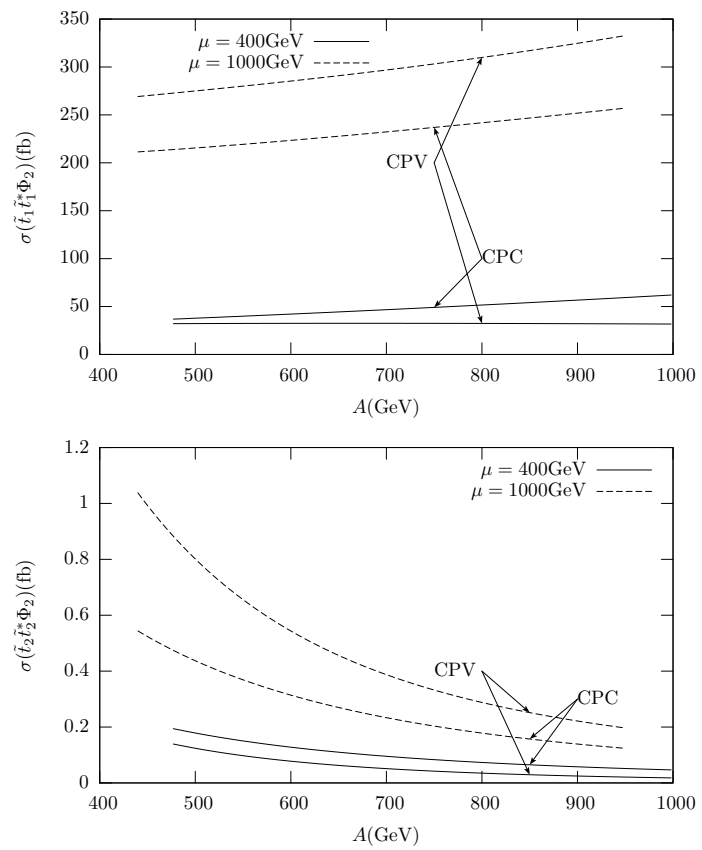

FIG. 7: The cross sections of $\Phi_{2}$ productions associated with squark pairs as the functions of $A$, where $\Phi_{2}=H^{0}$ in CPC and $\Phi_{2}=h_{2}$ in CPV, assuming $\tan \beta=5$ and $m_{\tilde{t}_{1}}=100 \mathrm{GeV}$. 